\title{
Determinants of Obesity and Metabolic Health in the Afghan Population: Protocol, Methodology and Preliminary Results
}

\section{Mohammad Sediq Sahrai}

International Agency for Research on Cancer https://orcid.org/0000-0002-3160-6502

Inge Huybrechts

International Agency for Research on Cancer

Carine Biessy

International Agency for Research on Cancer

Sabina Rinaldi

International Agency for Research on Cancer

Pietro Ferrari

International Agency for Research on Cancer

Abdul Wahed Wasiq

Kandahar University Faculty of Medicine

Marc J. Gunter

International Agency for Research on Cancer

Laure Dossus ( $\boldsymbol{\nabla}$ dossusi@iarc.fr)

International Agency for Research on Cancer https://orcid.org/0000-0003-2716-5748

\section{Study protocol}

Keywords: dietary patterns, obesity, metabolic health, Afghanistan

Posted Date: February 19th, 2020

DOI: https://doi.org/10.21203/rs.2.23970/v1

License: (c) (1) This work is licensed under a Creative Commons Attribution 4.0 International License.

Read Full License

Version of Record: A version of this preprint was published at Journal of Epidemiology and Global Health on January 7th, 2022. See the published version at https://doi.org/10.1007/s44197-021-00026-0. 


\section{Abstract}

Background: Non-communicable diseases (NCDs) cause more than $70 \%$ of deaths worldwide and share modifiable risk factors including obesity and metabolic abnormalities. Over the past 15 years many changes in lifestyle, dietary patterns, physical activity and socioeconomic status have been observed in the Afghan population. This study aims to investigate which specific lifestyle factors, dietary patterns and characteristics of Westernization are associated with an increased risk of being overweight or obese and with poor metabolic health in the Afghan population.

Methods: A population-based cross-sectional study was conducted where a total of 729 male and female participants were recruited. Face to face interviews and anthropometric measurements were conducted by trained health staff using standardized questionnaires which included information on sociodemographic and housing characteristics, income, occupation, ethnicity, personal and family medical history, stress, anthropometry, diet and physical activity. Bioelectric impedance analysis (BIA) was used to estimate body composition, including overall body fatness. Physical activity was measured by using the short version of the International Physical Activity Questionnaire (IPAQ). For a comprehensive assessment of dietary intake, a food frequency questionnaire (FFQ) specific to the Afghan population was developed which included all local food items relevant to the population. Lipid profile and fasting glucose were measured in a local laboratory. Biospecimens were collected using dried blood spots (DBS) and faecal occult blood test (FOBT) cards to perform microbiome and biomarker-based research.

Discussion: This is the first study which will assess dietary patterns, lifestyle factors and their association with obesity and metabolic health in Afghanistan. Such a study will aid the development of dietary and lifestyle guidelines in Afghanistan which will promote better health and educate people to make healthy food choices. The findings will also help in designing and implementing effective public health strategies to promote a healthy lifestyle and prevent the epidemic of overweight and obesity, and hence, reduce the burden of non-communicable diseases in the region.

\section{Introduction}

Non-communicable diseases (NCDs) are responsible for more than $70 \%$ of deaths worldwide and have shared modifiable risk factors such as unhealthy diet, physical inactivity, tobacco and alcohol use. Some of these risk factors lead to obesity and metabolic health abnormalities (1). Obesity is a major public health problem worldwide and its prevalence has almost tripled over the last 45 years (2). Major determinants of obesity are high energy intakes (in particular high energy dense foods) and low energy expenditures (3). In Low- and Middle-Income countries (LMICs) experiencing nutrition transition, including Afghanistan, there is often the co-existence of undernutrition and overnutrition across the life course which may lead to specific food and health patterns (4).

Over the last 15 years many changes have been observed in the lifestyle, dietary patterns, physical activity and socioeconomic status of the Afghan population. However, little is known on the specific 
determinants for obesity and poor metabolic health in Afghanistan. Due to rapid economic, social, and cultural changes in Afghanistan, dietary patterns are shifting from the traditional diets and lifestyles to more westernized habits with a marked increase in consumption of energy rich foods. As a consequence, the incidence of non-communicable diseases such as diabetes, cardiovascular disease and certain cancers is rising in Afghanistan where the burden of communicable diseases is already affecting the country (5).

To collect valuable data on the diet and lifestyle habits of the Afghan population and their association with obesity and metabolic parameters, we have conducted a population-based cross-sectional study in Kandahar city, called Kandahar Obesity Research (KOR). We hypothesize that specific lifestyle factors and dietary patterns, and characteristics of Westernization are associated with an increased risk of being overweight or obese and with poor metabolic health. To address this hypothesis, the overall objectives of the study are:

1. To develop a cross-sectional study focused on overweight, obesity and metabolic health

2. To identify determinants of obesity in this population

3. To inform public health strategies to promote a healthy lifestyle and prevent the epidemic of overweight and obesity, and hence, reduce the burden of non-communicable diseases in the region

To do so, we:

1. Collected information on socio-demographic and housing characteristics, income, occupation, ethnicity, personal and family medical history, stress, anthropometry, and physical activity

2. Collected biological specimens, such as blood, urine and stool

3. Will evaluate the associations of dietary patterns, physical activity and other lifestyle factors with various anthropometric measurements

4. Will evaluate the associations of nutritional and metabolic biomarkers with various anthropometric measurements

5. Will investigate the role of microbiome on obesity

To our knowledge, this is the first and largest population-based study conducted in Afghanistan on diet using a food frequency questionnaire and with the collection of biological specimens for future biomarker-based research.

\section{Methods}

\subsection{Study design:}

A population-based cross-sectional study was conducted in the medical research unit of Kandahar University in Kandahar, Afghanistan. The entire study recruitment was carried out in seven months from November, 2018 to May, 2019. The recruitment process for the survey was announced to the public by 
distributing study brochures in Kandahar University and to each study participant. These brochures included all the information about the study in the local language and instructions for registration. The eligible criteria were: 1) male or female residents of Kandahar province (living for more than one year), 2) aged between 20 and 75 years, 3) able to sign informed consent and provide bio-specimens. Inability to provide informed consent, pregnant women, people with severe health diseases and visitors from other provinces were excluded from the study. We used stratified sampling to recruit equal number of normal weight, overweight and obese participants. A total of 729 participants were recruited from the population residing in the province and after excluding underweight participants and those with missing nutritional assessment data, 711 were finally included in the study for statistical analyses.

\subsection{Sample size:}

Sample size calculations demonstrated that a total of 691 subjects would be sufficient to detect a correlation of at least 0.3 with $80 \%$ statistical power (type I error $5 \%$ ). Therefore, we will have $>80 \%$ power at a significance level of 0.05 to detect a correlation of 0.3 or higher between i.e. dietary intakes or biomarker levels and obesity in 711 subjects. We have $>80 \%$ power at a significance level of 0.05 to detect a correlation of 0.4 or higher between i.e. dietary intakes or biomarker levels and obesity on 355 subjects (when stratifying the analyses by gender) or on 178 subjects (when stratifying the analyses by gender and age). After correction for multiple testing (20 independent tests), we will still have $>80 \%$ to detect a 0.4 correlation in 711 and 355 subjects and to detect a 0.5 correlation in 178 subjects. The calculations have been performed with the 'Power' statistical procedure of SAS 9.4".

\subsection{Ethics:}

An informed consent form was developed in the Pashto language which included the information sheet and the consent certificate. This consent form, study protocol and all the questionnaires were submitted to the IARC Ethics Committee, Kandahar University's institutional review board (IRB) and FHI 360's IRB. A consent form was obtained from each participant. A project description leaflet was developed in Pashto language and given to all participants before their meeting with trained staff, which clearly explained the scope of the study, the extent of participation and the expected benefits of the study as a whole for better prevention of obesity.

\subsection{Measures and data collection:}

All the participants were provided with information about the study and were asked to provide written informed consent (in Pashto language). Face to face interviews and anthropometric measurements were conducted by trained health staff using standardized questionnaires. Training of health staff for interviews and study procedures was conducted in the medical faculty. Interviews and all the study procedures of the participants were undertaken in the medical research unit following Standard Operating Procedures (SOPs) specifically developed or adapted for the study. Table 1 summarises all the measurements of data collection in the study. 


\begin{tabular}{|c|c|c|}
\hline Examination & Measurements & Details \\
\hline \multirow{16}{*}{$\begin{array}{l}\text { Face to face } \\
\text { interview }\end{array}$} & Demographics & Sex, age, ethnicity, year of birth, address, housing characteristics, \\
\hline & and & education, marital status, occupation, household income \\
\hline & socioeconomic & \\
\hline & information & \\
\hline & Lifestyle & Smoking, tobacco snuff, alcohol, physical activity \\
\hline & Medical history & Cardiovascular, respiratory, digestive and urogenital diseases, cancer, \\
\hline & & diabetes, allergy, and oral health \\
\hline & Family history & High blood pressure, diabetes, cancer and others \\
\hline & of disease & \\
\hline & Reproductive & Age at menarche, age at menopause, contraception, reproductive \\
\hline & history & organs surgery, parity, and breastfeeding \\
\hline & (women) & \\
\hline & Stress & Anxiety and depression symptoms (Hopkins Symptoms Checklist) \\
\hline & Dietary groups & Dairy; meat, eggs and fish; fruits; vegetables; legumes; whole and \\
\hline & & refined cereals; nuts, seeds and dried fruits; fats and oil; fast food and \\
\hline & & snacks; sweets and desserts; beverages; spices and condiments \\
\hline Physical & Anthropometric & Weight, height, sitting height, body mass index (BMI), waist and hip \\
\hline Examination & measures & circumferences, blood pressure, imaging (liver ultrasound), bioelectric \\
\hline & & impedance analysis (BIA) \\
\hline Clinical lab- & Blood & Total cholesterol (TC), triglycerides (TG), high- and low-density \\
\hline based tests & & cholesterol lipoprotein (HDL-C, LDL-C), and fasting blood glucose \\
\hline Bio-sample & Blood, stool and & GenSaver, GenCollect, and BioSample TFN collection cards (Ahlstrom) \\
\hline collection & urine & to be stored at IARC for future biomarker analyses (i.e. metabolomics, \\
\hline & & inflammation markers, fatty acids, microbiome) \\
\hline
\end{tabular}


A standardized questionnaire was developed from other studies conducted in collaboration with IARC $(6,7)$ and administered by trained health workers in the research centre. It included information on sociodemographic and housing characteristics, income, occupation, ethnicity, personal and family medical history, stress, anthropometry, and physical activity. Socio-economic and demographic indicators include age, marital status, religion, number of siblings and children, education level, occupation, ethnicity (Pashtun, Tajik, Hazara, Uzbek, Baloch, others), residential address, type of housing, ownership of house and other house items. Personal and medical history include smoking status, diseases during childhood, vaccination history, previous diseases, present chronic diseases, trauma, anxiety and depression (using Hopkins Symptoms Checklist (HSCL)), previous hospitalization, recent treatment for a disease, regular intake of any medicine or dietary supplements, use of herbs and medicine plants, oral health, family history of cancer and other chronic disease in first degree relative, and for women, parity, age at first pregnancy, duration of breastfeeding, menstrual cycle history (age at menarche, menopausal status), use of oral contraceptive and hormone therapy.

Anthropometric measurements were performed by trained health personnel. Body weight was measured in all participants dressed in thin clothes with a digital electronic scale to the nearest $0.1 \mathrm{~kg}$. Both standing and sitting heights were measured without shoes with a stadiometer to the nearest millimetre. Body mass index (BMI) was calculated as weight (kilograms) divided by standing height (meters) squared. We used WHO's classification for BMI categories: normal weight (BMI of 18.5-24.9), overweight (BMI of 25.0-29.9) and obese (BMI of $\geq 30$ ). Waist circumference was measured midway between the lowest rib and superior border of the iliac crest at the end of normal expiration using a non-elastic tape to the nearest millimetre. Hip circumference was measured in standing position at the level of the most prominent part of the gluteus. Waist/hip ratio (WHR) was calculated from these measurements. For abdominal obesity, a cut-off point of $80 \mathrm{~cm}$ and $94 \mathrm{~cm}$ and for waist/hip ratio a cut-off point of 0.85 and 0.90 were considered for women and men, respectively.

Bioelectric impedance analysis (BIA) was used (Nutriguard Data Input device) to estimate body composition, particularly body fat. All the BIA measurements were performed while the person was lying in the supine position. To determine body fat (in kg and percent), lean mass (fat-free mass), body water, plus body cell mass (BCM) and extra-cellular mass (ECM), four electrodes were placed on the right hand, wrist, foot, and ankle and were connected to a generator applying an alternating electrical current of 0.8 $\mathrm{mA}$ and $50 \mathrm{kHz}$.

Participants were also asked about changes in their body weight during the last year, weight control methods and body silhouette at various ages. Pictograms with 9 options (from very thin to very fat), which are validated in different settings (8), were shown to both male and female participants to identify their body silhouette at different ages: Men, around ages 5 to 10 years, 15, 20,30, 40, 50 years and at current age; Women, around ages 5 to 10 years, at menarche, 15, 20, before first birth, 30, 40, 50 years, at menopause and at current age. 
Physical activity (PA) was measured by using the short version of International Physical Activity Questionnaire (IPAQ) (9). The questionnaire provided information about the duration of the physical activity in hours and minutes during a usual week. The intensity of physical activity was recorded as light, moderate and vigorous physical activities and later converted into METS (metabolic equivalent).

\section{Dietary Assessment:}

For a comprehensive assessment of dietary intake, a detailed country-specific food frequency questionnaire (FFQ) was developed which included all local food items relevant to the population. Data from previous dietary monitoring surveys have been used to select the food groups of interest (5). In addition, a research team of nutritional epidemiologists from IARC (International Agency for Research on Cancer) and Afghanistan checked the food list derived from these existing resources to optimize the food list and examples to be included further considering usual foods commonly consumed in Afghanistan. The food list of the final questionnaire consisted of different food items/categories (combining foods with similar food composition) and different consumption frequency categories (number of times per day, per week, per month, rarely or never). All foods consumed by the population in large and small quantity were included in the questionnaire. Different kinds of traditional and industrial beverages consumed by the population were also included in the questionnaire.

Each food item in the FFQ was assigned a portion size using standard local household units such as a plate, bowl, spoons of different sizes (tablespoon, teaspoon), tea-pot, tea-glass, and glass of water, as well as using photographs of foods and typical preparations of the local population, included in a food portion photograph book (FPPB) developed by the research team.

Regarding seasonal foods, participants were asked to answer the question based on intakes during periods/seasons when these foods were available. The daily intake was calculated according to the number of months per year that each seasonal food item was available.

The research team will also compile Food Composition Tables. For the compilation of the food composition tables we will use the indirect method based on pre-existing data from literature and local/neighbouring regions. Our data will be compiled according to international standards and guidelines for food composition data set by the FAO/INFOODS [FAO/INFOODS (2012). FAO/INFOODS Guidelines for Checking Food Composition Data prior to the Publication of a User Table/DatabaseVersion 1.0. FAO, Rome]. The food composition table will be used to assess nutrient intakes from the FFQ.

The dietary intake assessment was performed by trained health staff who asked the participants to report their habitual consumption frequency of the different foods listed in the FFQ during the last year. The food portion photograph book showed life-size colour photographs of foods in four portion sizes with photographs of utensils. The frequency categories included in the questionnaire were: Never, $<1$ time per month, 1-3 times a month, once/week, 2-4 times/week, 5-6 times/week, once/day, 2-3 times/day, 4-5 times/day and 6 times/day. 
Daily food intakes were calculated by multiplying the frequency of consumption with the selected portion size, considering also the number of months per year that seasonal foods were available.

\section{Clinical Non-invasive Assessment:}

As no incentive was paid to the participants, the following non-invasive clinical measures were used to facilitate recruitment and as a compensation for the participant's time and contribution. Blood pressure was measured by a mercury sphygmomanometer at right hand supported at heart level after sitting quietly for 15 minutes and recorded to the nearest $2 \mathrm{mmHg}$. Hypertension was defined as systolic blood pressure values of $\geq 140 \mathrm{mmHg}$ and/or diastolic blood pressure values of $\geq 90 \mathrm{mmHg}$ (10) or based on use of antihypertensive medicine. Abdominal ultrasound was performed by a trained physician. The purpose of the procedure was to scan the abdomen for any pathological changes. The liver was specifically scanned for the diagnosis of non-alcoholic fatty liver disease.

Participants were also evaluated for symptoms of anxiety and depression using Hopkins Symptoms Checklist (HSCL-25). This questionnaire contained 25 questions, where 10 and 15 questions were asked to assess anxiety and depression symptoms, respectively (11). A 4-point Likert scale was used to score each symptom and the total score was divided by 25 (total number of symptoms). Participants with a mean score of $\geq 1.55$ were considered probable psychiatric cases and those with a mean score of more or equal to standard cut-off of 1.75 were considered symptomatic (12).

The results of the study were shared with the participants and on their request, they were also provided with feedback about their diet, physical activity level and blood lipid and glucose profile. In case of any abnormal findings, the participants were examined free of cost and treated similarly to other patients by a specialist according to the facilities present in the teaching hospital.

\subsection{Biological Specimen Collection:}

IARC's standardized protocol for specimen collection was implemented as described in "Common Minimum Technical Standards and Protocols for Biobanks Dedicated to Cancer Research, IARC Technical Publication No. 44, 2017" (http://publications.iarc.fr/Book-And-Report-Series/larc-TechnicalPublications/Common-Minimum-Technical-Standards-And-Protocols-For-Biobanks-Dedicated-To-CancerResearch-2017)". These protocols have been extensively used in epidemiological studies led by IARC in various settings (13).

Dried blood spot (DBS), dried urine strip (DUS) and faecal occult blood test (FOBT) cards are an easy and inexpensive means of collection and storage of biospecimens in settings where collection and storage of plasma, urine and stool is not optimal (e.g. due to poverty, logistic or environmental/climate constraints). DBS can be used for molecular biology techniques and other diagnostic assays. All of these collection cards can reduce the cost and difficulty of cold chain shipping of samples and can be shipped as nondangerous goods at room temperature $(14,15)$. 
Blood samples were collected in TFN and GenSaver cards, stool samples were collected in GenSaver and GenCollect cards and urine samples were collected in GenCollect cards. 99.5\% of the participants provided with both types of blood cards. For GenCollect $82 \%$ and for GenSaver $83 \%$ of the participants provided stool samples. Urine cards were prepared only for the first hundred participants as a sample. Before sample collection the following data were recorded from all subjects: date and time of sample collection, date and time of preparing and packing biospecimen collection cards, fasting status (blood samples were only collected during fasting), time since last meal or drink, diet on previous day given that intake of some foods may affect biological measurements, smoking status, last menstrual cycle (women only), and use of any type of drugs or dietary supplements (i.e. multivitamins) in past week. All DBS, DUS and FOBT samples were stored in a clean, dry, temperature and humidity-controlled area of the laboratory, not exceeding $30^{\circ} \mathrm{C}$ and with no direct sunlight exposure before their shipment to IARC for storage and biomarker analyses.

Blood samples:

Blood samples were obtained by venipuncture from fasting participants seated in a semi upright position into two vacutainers tubes of $5 \mathrm{ml}$ containing heparin anticoagulant. One tube was analysed for lipid and glucose levels and the second tube was used for the preparation of DBS on one GenSaver and two BioSample TFN collecting cards (Ahlstrom). Using a pipette with a disposable tip, $125 \mu$ and $50 \mu$ of blood (for Gensaver and TFN respectively) was transferred by the laboratory technician to the centre of one circle on a labelled filter card without touching the filter paper directly with the tip of the pipette. This procedure was repeated to fill all circles of the cards and three cards were prepared per participant. The paper cards were then let dry for four hours at room temperature and later placed in a labelled plastic bag with a desiccant sachet with a humidity indicator. DBS were stored at room temperature before shipment to IARC.

Stool samples:

All stool samples were collected at the study recruitment site, using a standard protocol: stool collection paper was placed inside the toilet bowl. The participant collected a sample of the stool with a spatula, placed it in a labelled stool collection cup and returned the container to the laboratory. There, a small portion of stool was scraped by the laboratory technician using a wooden stick and all four cells of the card were smeared thinly with stool and the flap was closed. For each participant two stool cards, a GenSaver and a GenCollect, were collected. When completed, the stool cards were inserted into a gasimpermeable labelled plastic bag containing a desiccant pack with a humidity indicator.

Urine samples:

Participants provided urine samples in separate clean plastic containers along with stool samples. GenCollect urine card was soaked with urine in the container until it was completely saturated. The card was then suspended in a secure place and allowed to dry for around 6 hours. Finally, the dried card was covered with the flap and sealed in a labelled plastic bag with a humidity indicator desiccant pack. Two 
DUS cards were prepared for each participant. Before their shipment to IARC, these samples were kept in a temperature not exceeding $30^{\circ} \mathrm{C}$ and humidity-controlled (no more than $22 \%$ ) room, safe from direct exposure to sunlight.

Biomarker analyses to measure metabolic health:

Glucose and lipid profile (total cholesterol, triglyceride, HDL-cholesterol and LDL-cholesterol) measurements were performed in plasma from fasting participants in Kandahar University Teaching Hospital. Samples were centrifuged and analysed using an enzymatic photometric test method within one hour of sample collection. Participants with a fasting plasma glucose level of $>115 \mathrm{mg} / \mathrm{dL}$ or on antidiabetic medicine were considered diabetics. Dyslipidaemia was defined as triglycerides $>200 \mathrm{mg} / \mathrm{dL}$, total cholesterol $>200 \mathrm{mg} / \mathrm{dL}$, LDL-C $>130 \mathrm{mg} / \mathrm{dL}$ or $\mathrm{HDL}-\mathrm{C}<40 \mathrm{mg} / \mathrm{dL}(16-18)$.

Future biomarker analyses:

The establishment of a biobank, which includes DBS, DUS and dried stool samples will be of great value to perform high-quality biomarker-based research, such as metabolomics and microbiome analyses on stool and blood samples and DNA extraction from DBS for genetics and epigenetics studies.

All questionnaire data and biological samples were centralized at the International Agency for Research on Cancer in Lyon, France.

2.6. Data handling and statistical analysis:

Data cleaning

All data of the questionnaire were entered into Epi Info 7. The electronic version of the database was double-checked with paper forms which were completed manually during interviews. A unique numerical identifier was given to each study participant. Data variables were properly coded and labelled for statistical analyses. Duplicate observations and invalid data points were removed and spelling errors were corrected.

Statistical analyses

We will assess the association between demographic risk factors, dietary patterns and biological markers and metabolic health. All potential confounders available will be adjusted for in each analysis. Logistic regression and standard normal regression will be used to study the association between the exposures of interest and the risk of obesity. Statistical analyses are performed at Kandahar University and at IARC by STATA version 14, SPSS version 23 and SAS 9.4.

\section{Preliminary Results}

Major descriptive characteristics of the study population are presented in Table 2. A total of 711 subjects, 302 (42\%) women and 409 (58\%) men, were included in the analyses. As we used stratified sampling, 
approximately the same proportion of normal weight (36.2\%), overweight (32.8\%) and obese (31.0\%) participants were recruited. Obesity was however significantly higher in women (44.7\%) than in men (21\%). Mean age at recruitment was 37.4 years, higher for women than men (42 vs 34 ). A majority of the study population was 20 to 30 years of age (42.9\%), living in urban area $(92.3 \%)$, and married $(73.1 \%)$, whereas, half of the participants $(50.8 \%)$ were not educated. Mean BMI and waist circumference were $29.5 \mathrm{~kg} / \mathrm{m}^{2}$ and $97.3 \mathrm{~cm}$ among women, and were higher than men, $26.1 \mathrm{~kg} / \mathrm{m}^{2}$ and $93.7 \mathrm{~cm}$, respectively. $8.6 \%$ of the participants were tobacco smokers, higher in men $(13.2 \%)$ than in women $(2.3 \%)$, while tobacco snuff users were $14.8 \%$, also higher in men $(22.3 \%)$ than in women $(4.6 \%)$. Women were more active than men in moderate activity, while vigorous and light physical activities were higher among men.

Table 2 Major descriptive characteristics of the study population according to gender 


\begin{tabular}{|c|c|c|c|c|}
\hline \multirow[t]{3}{*}{ VARIABLES $^{1}$} & \multicolumn{2}{|c|}{ GENDER } & \multirow{3}{*}{$\begin{array}{l}\text { Total } \\
(\mathrm{n}=711)\end{array}$} & \multirow[t]{3}{*}{$\mathrm{P}$ value $^{2}$} \\
\hline & FEMALE & MALE & & \\
\hline & $302(42 \%)$ & 409 (58\%) & & \\
\hline \multicolumn{5}{|l|}{ Mean (STD) } \\
\hline Age (years) & $42.0(13.4)$ & $34.0(13.2)$ & $37.4(13.8)$ & $<0.001$ \\
\hline People in the house & $13.9(10.5)$ & $10.4(8.4)$ & $12(9)$ & $<0.001$ \\
\hline Weight (kg) & $71.2(15.4)$ & $76.6(16.1)$ & $74.3(16.0)$ & $<0.001$ \\
\hline Height $(\mathrm{cm})$ & $155.4(5.4)$ & $171.2(5.9)$ & $164.5(9.7)$ & $<0.001$ \\
\hline Sitting Height $(\mathrm{cm})$, missing $=1$ & $79.6(3.7)$ & $89.5(3.4)$ & $85.3(6.0)$ & $<0.001$ \\
\hline Waist circumference $(\mathrm{cm})$ & $97.3(14.9)$ & $93.7(14.5)$ & $95.2(14.8)$ & $<0.001$ \\
\hline Hip circumference $(\mathrm{cm})$ & $106.3(13.3)$ & $100.5(9.6)$ & $103.0(11.7)$ & $<0.001$ \\
\hline Body Mass Index $\left(\mathrm{kg} / \mathrm{m}^{2}\right)$ & $29.5(6.4)$ & $26.1(5.1)$ & $27.5(5.9)$ & $<0.001$ \\
\hline Waist to Hip ratio (WHR) & $0.91(0.07)$ & $0.93(0.08)$ & $0.92(0.08)$ & 0.016 \\
\hline \multicolumn{5}{|l|}{ Weekly Physical Activity } \\
\hline Vigorous Activity (METs) & $160.5(514)$ & 337.6 (1209) & $262.4(980)$ & 0.017 \\
\hline Moderate Activity (METs) & $1586.5(1577)$ & $376.3(958)$ & $890.3(1393)$ & $<0.001$ \\
\hline Light Activity (METs) & $292.5(556)$ & $696.1(841)$ & $525.6(760)$ & $<0.001$ \\
\hline Total Activity (METs) & 2039.5 (1780) & $1410.0(1829)$ & 1677.3 (1829) & $<0.001$ \\
\hline Daily Sitting Time (Hours) & $9.6(3)$ & $11.0(3)$ & $10.4(3)$ & $<0.001$ \\
\hline Daily Sleeping Time (Hours) & $8.2(1.6)$ & $7.4(1.3)$ & $7.7(1.5)$ & $<0.001$ \\
\hline Anxiety/Depression Score (HSCL-25) & $2.25(0.6)$ & $1.58(0.4)$ & $1.87(0.6)$ & $<0.001$ \\
\hline \multicolumn{5}{|l|}{ N (\%) } \\
\hline BMI Classes & & & & $<0.001$ \\
\hline Normal Weight & $78(25.8)$ & $179(43.8)$ & $257(36.2)$ & \\
\hline Overweight & 89 (29.5) & $144(35.2)$ & $233(32.8)$ & \\
\hline Obese & $135(44.7)$ & $86(21.0)$ & $221(31.0)$ & \\
\hline Age in Categories & & & & $<0.001$ \\
\hline $20-30$ & $76(25.1)$ & $229(56.0)$ & $305(42.9)$ & \\
\hline $31-40$ & $69(22.9)$ & 65 (15.9) & 134 (18.9) & \\
\hline $41-50$ & $91(30.1)$ & $62(15.2)$ & $153(21.5)$ & \\
\hline & Page $12 / 18$ & & & \\
\hline
\end{tabular}


Living Address

Rural

Urban

Marital Status

Married

Single

Widow/Separated

Educational Status

None

Up-to High School

Higher Education

Occupational Categories

Non-manual Workers

Farm Workers

Manual Workers

Students

Housewife/Jobless

Smoking status

Never Smoker

Former Smoker

Current Smoker

Snuffing status

Never

Former

Current

Central Obesity

No

Yes

Hypertension

19 (6.3)
46 (15.2)

256 (84.8)
27 (8.9)

$275(91.1)$

28 (6.9)

381 (93.1)

55 (7.7)

656 (92.3)

$<0.001$

$$
\begin{gathered}
241(79.8) \\
19(6.3) \\
42(13.9)
\end{gathered}
$$

$1(0.3)$

$<0.001$

269 (89.1)

$28(9.3)$

$5(1.7)$

$92(22.5)$

126 (30.8)

191 (46.7)

$<0.001$

$\begin{array}{ccc}4(1.3) & 150(36.7) & 154(21.7) \\ 0(0) & 20(4.9) & 20(2.8) \\ 1(0.3) & 63(15.4) & 64(9.0) \\ 3(1) & 148(36.2) & 151(21.2) \\ 294(97.4) & 28(6.8) & 322(45.3)\end{array}$

276 (92.4)

285 (69.7)

561 (78.9)

70 (17.1)

$89(12.5)$

7 (2.3)

54 (13.2)

61 (8.6)

$<0.001$

285 (94.4)

306 (74.8)

$3(1.0)$

12 (2.9)

$14(4.6)$

91 (22.3)
212 (51.8)

$197(48.2)$
591 (83.1)

$15(2.1)$

105 (14.8)

$<0.001$ $<0.001$

258 (36.3)

453 (63.7) 

No
$143(47.4)$
309 (75.6)
452 (63.6)
Yes
159 (52.6)
$100(24.4)$
259 (36.4)

Diabetes Mellitus

0.07

No

243 (80.5)

350 (85.6)

593 (83.4)

Yes

59 (19.5)

59 (14.4)

118 (16.6)

Dyslipidaemia

0.001

No

$152(50.3)$

256 (62.6)

$408(57.4)$

Yes

150 (49.7)

153 (37.4)

303 (42.6)

Fatty Liver $<0.001$

No

$166(55.0)$

$288(70.4)$

454 (63.9)

Yes

$136(45.0)$

121 (29.6)

257 (36.1)

Anxiety/Depression (HSCL-25)

$<0.001$

No

$29(9.6)$

208 (50.9)

237 (33.3)

Probable

32 (10.6)

88 (21.5)

120 (16.9)

Case

241 (79.8)

$113(27.6)$

$354(49.8)$

1 Number of missing values is 0 unless otherwise specified; ${ }^{2}$ Chi-square test or ANOVA

Continuous variables are shown with mean and standard deviation; Categorical variables are shown with number and percentage

Parameters of metabolic health and stress were higher in women than in men. Among women, the prevalence of central obesity, hypertension, diabetes mellitus, and dyslipidaemia was $84.8 \%, 52.6 \%$, $19.5 \%$ and $49.7 \%$, while in men it was $48.2 \%, 24.4 \%, 14.4 \%$ and $37.4 \%$, respectively. Mean score of Hopkins Symptoms Checklist (HSCL-25) was 1.87 for both sexes. However, the mean score was 2.25 for women and 1.58 for men. Almost half the participants (49.8\%) were symptomatic for anxiety/depression and it was significantly higher in women $(79.8 \%)$ than in men $(27.6 \%)$.

\section{Discussion}

This is the first population-based study which will focus on dietary patterns, demographic and lifestyle factors and their association with obesity and metabolic health in Afghanistan through collection of dietary data and biological samples. Such a study will aid the development of dietary and lifestyle guidelines in Afghanistan which will promote better health and educate people to make healthier food choices. 
NCDs are becoming one of the major health issues in a country with more than three decades of war, instability and widespread poverty. Overall, NCDs account for $44 \%$ of all deaths in the country (19). Therefore, NCDs and their modifiable risk factors should be tackled effectively. Meanwhile, obesity is associated with chronic diseases, such as stroke, hypertension, cardiovascular disease, type-2 diabetes, and certain forms of cancer. Obesity, especially when it is ignored and becomes chronic, is not only a problem of individuals, but it is a problem which affects the whole population and even generations. Obesity not only increases the risk of metabolic and chronic diseases, but it also impacts quality of life (20). Considering recent advances in nutrition science, focusing on diet can be helpful for the prevention of chronic diseases (21).

Through the establishment of a cross-sectional study in Kandahar city, one of the largest provinces of Afghanistan, we will examine the role of different factors such as dietary patterns, physical activity and lifestyle in the development of obesity and metabolic health. The findings will help in designing and implementing effective public health strategies to promote a healthy lifestyle and prevent the epidemic of overweight and obesity, and hence, reduce the burden of non-communicable diseases in the region.

Furthermore, the establishment of a large high-quality cross-sectional study in Afghanistan has great potential and is an important investment for future research hypotheses. The data collected will constitute an invaluable resource for future studies on lifestyle patterns, biomarkers and the microbiome, and could be used to train future epidemiologists and public health scientists in Afghanistan and other regions/countries with similar living conditions.

\section{Declarations}

\section{Acknowledgments}

The authors thank all the study participants, data collectors, technical staff and the academic staff of Afghanistan National Agricultural Sciences and Technology University (ANASTU): Abdullah Baryal, Halima Rashad, Azad Afghan, Khalid Akbari, Sayed Shah, Mohammad Tahir Motwahir, Rashid Ahmad Sahrai, Naqibullah Mojadidi, Mohammad Musa Daudzai, Sultan Mohammad Sirat, and Noorulhaq Mohammadi.

\section{Authors' Contributions}

LD, AWW and MJG: study concept, design and supervision. MSS, IH, CB, PF and LD: methodology, data analysis and interpretation. MSS and LD: data collection and drafting the manuscript. MSS, IH, SR, CB, $P F, M J G, A W W$ and LD: revision and editing of the manuscript. All authors have read and approved the final manuscript.

\section{Funding}

Mohammad Sediq Sahrai is the recipient of the University Support \& Workforce Development Program (USWDP) scholarship funded by the United State Agency for International Development (USAID). 
Availability of data and materials

The datasets used and/or analysed during the current study are available from the corresponding author on reasonable request.

\section{Ethics approval and consent to participate}

The research project was approved by the Institutional Review Board at Kandahar University. Written informed consent was obtained from all participants.

\section{Consent for publication}

Not applicable.

\section{Competing interests}

The authors declare that they have no competing interests.

\section{Disclaimer:}

Where authors are identified as personnel of the International Agency for Research on Cancer / World Health Organization, the authors alone are responsible for the views expressed in this article and they do not necessarily represent the decisions, policy or views of the International Agency for Research on Cancer / World Health Organization.

\section{References}

1. Riley L, Gouda H, Cowan M, World Health Organization. Noncommunicable diseases progress monitor, 2017 [Internet]. 2017 [cited 2020 Feb 10]. Available from:

http://apps.who.int/iris/bitstream/10665/258940/1/9789241513029-eng.pdf

2. Obesity and overweight [Internet]. [cited 2020 Feb 10]. Available from: https://www.who.int/newsroom/fact-sheets/detail/obesity-and-overweight

3. Ba S, I C, Jc S, Wpt J. Diet, nutrition and the prevention of excess weight gain and obesity. Public Health Nutr [Internet]. 2004 Feb [cited 2020 Feb 10];7(1a):123-46. Available from: https://www.cambridge.org/core/product/identifier/S1368980004000175/type/journal_article

4. On behalf of the IARC working group on Energy Balance and Obesity, Romieu I, Dossus L, Barquera S, Blottière HM, Franks PW, et al. Energy balance and obesity: what are the main drivers? Cancer Causes Control [Internet]. 2017 Mar [cited 2020 Feb 10];28(3):247-58. Available from: http://link.springer.com/10.1007/s10552-017-0869-z

5. UNICEF. National nutrition survey: Afghanistan (2013). Kabul U N Child Fund Retrieved Httpsreliefweb Intsitesreliefweb IntfilesresourcesReport 20NNS 20Afghanistan. 2014;202013(20):2026-14. 
6. Beasley JM, Coronado GD, Livaudais J, Angeles-Llerenas A, Ortega-Olvera C, Romieu I, et al. Alcohol and risk of breast cancer in Mexican women. Cancer Causes Control [Internet]. 2010 Jun [cited 2020 Feb 10];21(6):863-70. Available from: http://link.springer.com/10.1007/s10552-010-9513-x

7. Pourshams A, Khademi H, Malekshah AF, Islami F, Nouraei M, Sadjadi AR, et al. Cohort Profile: The Golestan Cohort Study-a prospective study of oesophageal cancer in northern Iran. Int J Epidemiol [Internet]. 2010 Feb 1 [cited 2020 Feb 10];39(1):52-9. Available from: https://academic.oup.com/ije/article-lookup/doi/10.1093/ije/dyp161

8. Tehard B. Anthropometric measurements and body silhouette of women: validity and perception. :10.

9. Craig CL, Marshall AL, Sj??Str??M M, Bauman AE, Booth ML, Ainsworth BE, et al. International Physical Activity Questionnaire: 12-Country Reliability and Validity: Med Sci Sports Exerc [Internet]. 2003 Aug [cited 2020 Feb 10];35(8):1381-95. Available from: https://insights.ovid.com/crossref? an $=00005768-200308000-00020$

10. Mancia G, Rosei EA, Azizi M, Burnier M, Clement DL, Coca A, et al. 2018 ESC/ESH Guidelines for the management of arterial hypertension. 2018;98.

11. Derogatis LR, Lipman RS, Rickels K, Uhlenhuth EH, Covi L. The Hopkins Symptom Checklist (HSCL): A self-report symptom inventory. Behav Sci [Internet]. 1974 Jan [cited 2020 Feb 10];19(1):1-15. Available from: http://doi.wiley.com/10.1002/bs.3830190102

12. Nettelbladt P, Hansson L, Stefansson C-G, Borgquist L, Nordstr $>m$ G. Test characteristics of the Hopkins Symptom Check List-25 (HSCL-25) in Sweden, using the Present State Examination (PSE-9) as a caseness criterion. Soc Psychiatry Psychiatr Epidemiol [Internet]. 1993 [cited 2020 Feb 10];28(3):130-3. Available from: http://link.springer.com/10.1007/BF00801743

13. Vaught JB, Caboux E, Hainaut P. International Efforts to Develop Biospecimen Best Practices. Cancer Epidemiol Biomarkers Prev [Internet]. 2010 Apr 1 [cited 2020 Feb 10];19(4):912-5. Available from: http://cebp.aacrjournals.org/cgi/doi/10.1158/1055-9965.EPI-10-0058

14. Holen T, Norheim F, Gundersen TE, Mitry P, Linseisen J, Iversen PO, et al. Biomarkers for nutrient intake with focus on alternative sampling techniques. Genes Nutr [Internet]. 2016 Dec [cited 2020 Feb 10];11(1):12. Available from: http://genesandnutrition.biomedcentral.com/articles/10.1186/s12263016-0527-1

15. Wang Z, Zolnik CP, Qiu Y, Usyk M, Wang T, Strickler HD, et al. Comparison of Fecal Collection Methods for Microbiome and Metabolomics Studies. Front Cell Infect Microbiol [Internet]. 2018 Aug 28 [cited 2020 Feb 10];8:301. Available from: https://www.frontiersin.org/article/10.3389/fcimb.2018.00301/full

16. Thomas L. Clinical laboratory diagnostics: Use and assessment of clinical laboratory results. THbooks Verlagsgesellschaft; 1998.

17. Schaefer EJ, McNamara JR. Overview of the diagnosis and treatment of lipid disorders. Handb Lipoprotein Test. 1997;25-48.

18. Detection NCEP (US) EP on, Adults T of HBC in. Third report of the National Cholesterol Education Program (NCEP) expert panel on detection, evaluation, and treatment of high blood cholesterol in 
adults (adult treatment panel III). National Cholesterol Education Program, National Heart, Lung, and Blood ...; 2002.

19. WHO | Noncommunicable diseases country profiles 2018 [Internet]. [cited 2020 Feb 10]. Available from: https://www.who.int/nmh/publications/ncd-profiles-2018/en/

20. Blüher M. Obesity: global epidemiology and pathogenesis. Nat Rev Endocrinol [Internet]. 2019 May [cited 2020 Feb 10];15(5):288-98. Available from: http://www.nature.com/articles/s41574-019-01768

21. Mozaffarian D. Dairy Foods, Obesity, and Metabolic Health: The Role of the Food Matrix Compared with Single Nutrients. Adv Nutr [Internet]. 2019 Sep 1 [cited 2020 Feb 10];10(5):917S-923S. Available from: https://academic.oup.com/advances/article/10/5/917S/5569504 